\title{
UTILIZAÇÃO DE RESÍDUOS DA MADEIRA DE Pinus spp. COMO SUBSTITUIÇÃO AO AGREGADO MIÚDO NA PRODUÇÃO DE BLOCOS DE CONCRETO PARA ALVENARIA ESTRUTURAL
}

\author{
USE OF WOOD RESIDUES OF Pinus spp. FOR SUBSTITUTION TO THE FINE AGGREGATE IN \\ THE PRODUCTION OF CONCRETE BLOCKS FOR STRUCTURAL MASONRY
}

\author{
Adauto José Miranda de Lima ${ }^{1}$ Setsuo Iwakiri²
}

\begin{abstract}
RESUMO
Este trabalho teve como objetivo avaliar o potencial de utilização do resíduo do desdobro de toras de Pinus spp. em substituição ao agregado miúdo do concreto traço 1:10, utilizado para a produção de blocos para alvenaria estrutural. A avaliação das características físicas (massa específica, índice de vazios e absorção de água) e mecânicas (resistência à compressão e à tração) do concreto foi realizada conforme normas da ABNT. Foram testadas substituições do agregado miúdo por teores de 0, 20, 40, 60, 80 e 100\% de resíduo de Pinus spp. in natura, Pinus spp. in natura e com adição de $4,5 \%$ de $\mathrm{CaCl}_{2} \cdot 2 \mathrm{H}_{2} \mathrm{O}$ e resíduo de Pinus spp. pré-tratado com extração em água fria por 48 horas (AF48H). As composições foram inicialmente utilizadas para a produção em laboratório, de corpos de prova cilíndricos de $50 \mathrm{~mm}$, com o uso de um sistema de moldagem por vibrocompressão e posteriormente, para produção de blocos, com os traços otimizados, em máquina vibroprensa. Obtiveram-se resultados satisfatórios com substituições de até $50 \%$ do agregado miúdo mineral pelo resíduo de Pinus spp.
\end{abstract}

Palavras-chave: resíduo de Pinus spp.; blocos de concreto; alvenaria estrutural.

\begin{abstract}
This research was developed with the objective of evaluating the potential use of residues of the production of Pinus spp. sawnwood, as substitution to the fine aggregate of the concrete used for the production of blocks for structural masonry. The evaluation of the physical characteristics (density, porosity and water absorption) and mechanics (compression and tension strength) of the concrete was evaluated in agreement with the ABNT normalization. Substitutions of the fine aggregate were tested by percentages of 0,20 , 40, 60, 80 and $100 \%$ of residue of Pinus spp. in natura, Pinus spp. in natura and with addition of 4,5\% $\mathrm{CaCl}_{2} \cdot 2 \mathrm{H}_{2} \mathrm{O}$ and residue of Pinus spp. pre-treated with extraction in cold water for 48 hours (AF- $\left.48 \mathrm{H}\right)$. The compositions were initially used for the production, in laboratory, of cylindrical specimens of $50 \mathrm{~mm}$ diameter, by the use of a molding system for vibrate-compression and later, for producing blocks, with optimized compositions, in vibrate-press commercial machine. Satisfactory results were obtained with substitutions of up to $50 \%$ of the mineral fine aggregate for the residue of Pinus spp.
\end{abstract}

Keywords: residue of Pinus spp., concrete blocks, structural masonry.

1 Engenheiro Florestal, Dr., Professor do Departamento de Construção Civil, Universidade Tecnologia Federal do Paraná, Rua Dep. Heitor de Alencar Furtado, 4900, CEP 81280-340, Curitiba (PR), Brasil. adautojm@utfpr.edu.br

2 Engenheiro Florestal, Dr., Professor do Departamento de Engenharia e Tecnologia Florestal, Universidade Federal do Paraná, Av. LothárioMeissner, 632, CEP 80210-170, Curitiba (PR), Brasil. setsuo@ufpr.br

Recebido para publicação em 12/04/2010 e aceito em 18/09/2012 


\section{INTRODUÇÃO}

A viabilidade da produção de compósitos produzidos com fibras ou partículas naturais vem sendo pesquisada há décadas, possuindo uma longa história de aceitação e aplicação na construção civil, especialmente na Europa e Ásia (IWAKIRI, 2005).

De acordo com Moslemi (1989) e Semple e Evans (2004), os materiais produzidos com compósito cimento-madeira apresentam uma série de vantagens, entre as quais podem ser citadas: disponibilidade de matéria-prima em grandes quantidades, baixa massa específica, baixa permeabilidade, possibilidade de serem serrados e de receberem parafusos e acabamentos, o que permite sua utilização na produção de painéis de fechamento, forros, telhas, elementos de vedação e de alvenaria.

Além dessas vantagens, os materiais baseados em compósitos cimento-madeira não emitem resíduos tóxicos durante sua manufatura, não necessitam de tratamentos preservativos (RAMIREZCORETTI et al., 1998), apresentam menor dispêndio de energia no processo de produção, inclusive se tornando uma alternativa viável aos fibrocimentos que utilizam asbesto (amianto), principalmente na Europa, Japão e América do Norte (MOSLEMI, 1989), além do que apresentam boas características como isolante termoacústico (SAVASTANO JÚNIOR e AGOPYAN, 1997), excelente estabilidade dimensional (JORGE et al., 2004) e grande capacidade de dissipação de energia (SEMPLE e EVANS, 2004).

A principal desvantagem a ser citada, conforme Hachmi e Campbell (1989) e Moslemi (1989), diz respeito à incompatibilidade química entre o cimento e a madeira. A constituição química da madeira, em função da presença de açúcares, resinas, fenóis e amido, pode afetar de forma adversa as reações de hidratação do cimento Portland, fato que, conforme Simatupang et al. (1988), pode ser melhorado com tratamentos simples e confiáveis, tais como extração em água quente ou fria.

O gênero Pinus, da família das Pinaceae, é composto por plantas lenhosas, em geral arbóreas, de altura variável, sendo que algumas espécies de Pinus se incluem entre as árvores mais utilizadas no mundo (LIMA et al., 1988; MORAIS et al., 2005).

As madeiras pertencentes ao gênero Pinus fornecem material de boa qualidade para a produção de celulose, além de apresentarem características tecnológicas adequadas para seu aproveitamen- to em serraria. Conforme Fagundes (2003), durante as etapas do processamento mecânico da madeira produzse grande quantidade de resíduos que não têm recebido a devida valorização.

$\mathrm{O}$ incremento na produção de madeira de Pinus spp. pode ser verificado com base na análise dos dados da Associação Brasileira da Indústria de Madeira Processada Mecanicamente (ABIMCI, 2009), onde se verifica uma produção de toras de Pinus spp., em 2007, de 50,6 milhões de metros cúbicos, contra 42 milhões de metros cúbicos em 1997. Portanto, um acréscimo de produção de 20,48\% no período de dez anos.

Sob esta ótica, a geração de resíduos florestais no Brasil também assume valores expressivos, pois, segundo a ABIMICI (2009), só a produção de madeira serrada em 2008 alcançou o montante de 22.886 milhões de metros cúbicos. Inferindo sobre este valor, o potencial de perdas, que, conforme Freitas (2000), pode atingir até $60 \%$, deduz-se que um valor significativo de resíduos florestais foi produzido naquele ano, sem um efetivo reaproveitamento em outros produtos que possam prolongar o armazenamento do $\mathrm{CO}_{2}$ incorporado à madeira.

De acordo com Hon e Shiraiashi (2001), há um grande interesse da indústria da construção civil quanto ao reaproveitamento desses resíduos, pois a sua utilização vem se tornando uma questão de relevante importância econômica e ambiental e este reaproveitamento será uma maneira de resguardar os recursos naturais e reduzir a emissão de dióxido de carbono.

A norma brasileira NBR 6.136 (ABNT, 2008) define blocos vazados para alvenaria como sendo elementos que possuem furos prismáticos perpendiculares à face que os contém e cuja seção transversal média útil seja inferior a $75 \%$ da seção transversal bruta.

Os blocos de concreto, assim como são conhecidos hoje, foram desenvolvidos nos Estados Unidos da América, no final do século XIX. Acredita-se que o primeiro bloco de concreto tenha sido moldado nos EUA, em 1882 (MEDEIROS, 1993). No Brasil existem registros da utilização de blocos de concreto por volta de 1940, sendo que inúmeros edifícios vêm sendo construídos com este material (SOUSA, 2001). Conforme Sousa (2001), os blocos de concreto são materiais de grande interesse, além do custo relativamente baixo, apresentam também um baixo teor energético incorporado à sua produção, pois não passam pelo processo de queima, como os blocos cerâmicos para alvenaria. 
Isaia (2007) demonstra os diferenciais de um sistema construtivo tradicional, com blocos cerâmicos, para outro utilizando blocos de concreto, sendo que este último apresenta diversas vantagens em detrimento aos métodos convencionais, tais como: (a) redução do custo da obra de até $30 \%$; (b) maior velocidade na conclusão da obra; (c) menor custo para instalações elétricas e hidráulicas, uma vez que não há necessidade de quebrar as paredes para realizar estas instalações; (d) diminuição da necessidade de armaduras de aço e, consequentemente, de mão de obra e, no caso de alvenaria estrutural, dispensa o uso de vigas e pilares de concreto armado.

Normalmente, os materiais utilizados na produção de blocos de concreto resumem-se em aglomerante, agregados graúdos e miúdos, água e, eventualmente, aditivos e corantes.

De acordo com Ferreira Júnior (1985), o termo dosagem refere-se ao estudo realizado para fixar as quantidades dos materiais constituintes, visando obter um concreto e, consequentemente, os blocos pré-fabricados, com as características desejadas, que são: coesão no estado fresco, de forma que possam ser desmoldados e transportados sem que seu formato seja alterado; máxima compacidade, para que a absorção de água seja mínima e a resistência compatível com a aplicação a que se destina.

Neste contexto, o objetivo do trabalho foi desenvolver um novo tipo de bloco de concreto, incorporando o resíduo de Pinus spp. em substituição parcial ao agregado miúdo mineral (areia) e com características físicas e mecânicas que possibilitem a sua utilização em alvenaria estrutural.

\section{MATERIAIS E MÉTODOS}

\section{Materiais utilizados}

O resíduo de madeira Pinus spp. utilizado neste estudo, caracterizado como serragem, é proveniente do desdobro de toras de Pinus, de espécies e idades variadas, pela empresa Madeireira Juruqui, localizada no município de Almirante Tamandaré - PR. Após a coleta, a serragem foi seca ao ar e peneirada em uma peneira $A B N T n^{\circ} 4$, de forma a garantir a ela uma granulometria com partículas menores (Dmáx) que 4,8 mm.

$\mathrm{O}$ aditivo acelerador de pega utilizado foi o cloreto de cálcio bi-hidratado $\left(\mathrm{CaCl}_{2} \cdot 2 \mathrm{H}_{2} \mathrm{O}\right)$. $\mathrm{O}$ aglomerante utilizado na pesquisa foi o cimento
Portland, tipo CPV ARI RS - cimento Portland de Alta Resistência Inicial - Resistente a Sulfatos, especificado pela norma ABNT NBR 5.733 (ABNT, 1991).

As características do resíduo de Pinus spp. e dos agregados minerais convencionais utilizados, são apresentadas na Tabela 1 .

\section{Metodologia}

Em um estudo preliminar, foram produzidos três séries de blocos de concreto, com traços $1: 6,1: 8$ e $1: 10$. Como todos apresentaram desempenho satisfatório para utilização em alvenaria estrutural, ficou definido, principalmente em função das características econômicas, o traço 1:10 como referência para este estudo.

Os blocos de concreto foram produzidos em uma máquina vibroprensa marca Trillor Montana, modelo MBX 200. Este equipamento aplica uma carga unidirecional vertical de $1.010 \mathrm{kgf}$ durante a compressão do concreto fresco para produção dos blocos, sendo capaz de produzir até 4 mil blocos por dia.

A proporção do agregado total (agregado miúdo + agregado graúdo) foi feita mediante a determinação da mistura de maior massa unitária compactada, o que traduz o menor índice de vazios possível para a mistura, conforme método proposto por Ferreira Júnior (1985), e a umidade de moldagem, após testes de moldagem com a máquina produtora de blocos, foi fixada em teores entre $6 \%$ e $6,5 \%$ (em relação à soma das massas dos materiais secos, inclusive do cimento Portland). A composição do concreto traço $1: 10$ ficou estabelecida conforme Tabela 2.

$\mathrm{Na}$ produção dos concretos com substituição parcial do agregado miúdo convencional, a areia foi substituída por teores de $0,20,40,60$, 80 e $100 \%$ de resíduo de Pinus spp. in natura, ou seja, sem qualquer pré-tratamento ou uso de aditivos químicos; Pinus spp. in natura com $4,5 \%$ de $\mathrm{CaCl}_{2} \cdot 2 \mathrm{H}_{2} \mathrm{O}$; e Pinus spp. com pré-tratamento tipo extração em água fria por 48 horas $(\mathrm{AF} 48 \mathrm{H})$ e com $4,5 \%$ de $\mathrm{CaCl}_{2} .2 \mathrm{H}_{2} \mathrm{O}$.

A mistura dos materiais foi efetuada em uma betoneira de eixo inclinado com capacidade de 120 litros. O teor de água da mistura foi controlado e corrigido em função do teor de umidade do resíduo de Pinus spp., de forma a manter sempre a madeira no ponto de saturação das fibras (PSF), adotado neste estudo como $30 \%$. 
TABELA 1: Características do resíduo de Pinus spp. e dos agregados minerais convencionais.

TABLE 1: Characteristics of the residues of Pinus spp. and the conventional mineral aggregates.

\begin{tabular}{|c|c|c|c|c|}
\hline Características & Norma & $\begin{array}{l}\text { Agregado } \\
\text { Miúdo }\end{array}$ & $\begin{array}{l}\text { Agregado } \\
\text { Graúdo }\end{array}$ & $\begin{array}{l}\text { Resíduo de } \\
\text { Pinus spp. }\end{array}$ \\
\hline Massa específica $\left(\mathrm{g} / \mathrm{cm}^{3}\right)$ & $\begin{array}{c}\text { NBR NM } 52 \text { (ABNT, 2003) e } 53 \\
(\text { ABCP, 2003) }\end{array}$ & 2,64 & 2,70 & 1,28 \\
\hline Massa unitária $\left(\mathrm{g} / \mathrm{cm}^{3}\right)$ & $\begin{array}{c}\text { NBR NM } 52 \text { (ABNT, 2003) e } 53 \\
(A B C P, 2003)\end{array}$ & 1,56 & 1,52 & 0,17 \\
\hline $\begin{array}{l}\text { Densidade madeira sólida } \\
\left(\mathrm{g} / \mathrm{cm}^{3}\right)\end{array}$ & NBR 7190 (ABCP, 1997) & & & 0,41 \\
\hline Dimensão máxima (mm) & NBR NM 248 (ABCP, 2003) & 4,80 & 9,50 & 4,80 \\
\hline Módulo de finura & NBR NM 248 (ABCP, 2003) & 2,92 & 5,67 & 3,25 \\
\hline $\begin{array}{l}\text { Teor mat. pulverulento } \\
(\%)\end{array}$ & NBR NM 46 (ABCP, 2003) & 1,08 & 0,25 & 0,27 \\
\hline Agregado miúdo (\%) & NBR NM 248 (ABCP, 2003) & 96,82 & 7,05 & 99,73 \\
\hline Agregado graúdo (\%) & NBR NM 248 (ABCP, 2003) & 2,10 & 92,70 & 0,00 \\
\hline Teor argila em torrões (\%) & NBR NM 7218 (ABCP, 1987) & 0,00 & 0,00 & 0,00 \\
\hline Teor mat. orgânica (ppm) & NBR NM 49 (ABCP, 2001) & $<100,00$ & $<100,00$ & - \\
\hline Absorção de água (\%) & NBR NM 30 (ABCP, 2001) & 1,47 & 0,80 & 129,62 \\
\hline Coef. inchamento médio & NBR NM 6467 (ABCP, 2006) & 1,44 & - & 7,79 \\
\hline Teor umidade crítica (\%) & NBR NM 6467 (ABCP, 2008) & 5,00 & - & - \\
\hline Extrativos Totais (\%) & TAPPI T 204cm (1997) & - & - & 2,42 \\
\hline $\mathrm{pH}$ & TAPPI T 204cm (1997) & - & - & 7,79 \\
\hline Classificação & NBR 7211 (ABCP, 2009) & $\begin{array}{c}\text { Areia } \\
\text { Zona - } \\
3 \text { ABNT }\end{array}$ & Brita 0 & $\begin{array}{c}\text { Equivalente } \\
\text { Zona - } \\
3 \text { ABNT }\end{array}$ \\
\hline
\end{tabular}

TABELA 2: Composição para o concreto traço 1:10 em massa ( $1 \mathrm{~m}^{3}$ de concreto).

TABLE 2: Composition for concrete 1:10 in mass $\left(1 \mathrm{~m}^{3}\right.$ of concrete).

\begin{tabular}{cc}
\hline Traço em volume & $1: 10$ \\
\hline Material & Massa $(\mathrm{kg})$ \\
\hline Cimento & 220,00 \\
Agregado total & $2.119,10$ \\
Areia $-50 \%$ & $1.073,31$ \\
Brita $0-50 \%$ & $1.045,79$ \\
Água - umidade de & - \\
moldagem & \\
$\mathrm{U}=6,0 \%$ & 140,35 \\
\hline
\end{tabular}

A determinação das características físicas e mecânicas do concreto com as substituições parciais do agregado miúdo convencional pelo resíduo de Pinus spp., foi realizada em corpos de prova (CP) cilíndricos de concreto, com diâmetro nominal de $50 \mathrm{~mm}$, moldados em uma única camada e adensados pelo efeito concomitante de vibração e compressão, de forma a reproduzir a moldagem efetuada em uma máquina produtora de blocos (vibroprensa).

Para tanto foi utilizado um aparato baseado na norma ASTM C 1170 (2008). Segundo esta norma, a consistência do concreto é estabelecida em função do tempo que uma determinada amostra de concreto leva para adensar, sob determinada energia de adensamento, em uma mesa vibratória. Este tempo equivalente de moldagem, expresso em segundos, é denominado pela norma como tempo "Vebe" e foi definido para este estudo, com base nas características dos materiais e equipamentos utilizados em 27 segundos. 
Para a desmoldagem e cura do concreto produzido com os corpos de prova cilíndricos, seguiram-se os procedimentos indicados na NBR 5.738 (ABNT, 2003), e para determinação das características físicas e mecânicas, seguiram-se as normas NBR 7.215 (ABNT, 1997) e NBR 9.778 (ABNT, 2005).

A cura inicial dos corpos de prova, com duração de 24 horas, deu-se em câmara úmida, conforme NBR 9.479 (ABNT, 2006), com temperatura de $23 \pm 2{ }^{\circ} \mathrm{C}$ e umidade relativa mínima de $95 \%$, onde permaneceram com os moldes e cobertos com uma placa de acrílico, para proteção, conforme NBR 7.215 (ABNT, 1997). Após o período de cura inicial, os corpos de prova foram desmoldados, identificados e mantidos em câmara úmida, imersos em água, até a realização do ensaio.

As características mecânicas, compreendendo os ensaios para determinação da resistência à compressão aos sete dias (RC $7 \mathrm{~d})$ foram efetuadas conforme NBR 7.215 (ABNT, 1997). Para não haver influências de irregularidades nos topos dos corpos de prova, e garantir a ruptura por compressão simples, foram utilizados discos de neoprene com dureza 60 a 70 shore dispostos em guarnições de aço. $\mathrm{O}$ equipamento utilizado foi uma máquina universal de ensaio marca Emic, modelo DL 30000, com aquisição de dados computadorizada. As características físicas foram determinadas conforme norma NBR 9.778 (ABNT, 2005).

Após determinadas as composições tecnicamente viáveis de substituição do agregado miúdo mineral convencional pelos três tipos de resíduo de Pinus spp., realizou-se a produção dos blocos em fábrica. Após moldados, os blocos sofreram cura ao ar, com molhagens diárias, conforme o esquema de fabricação normal da fábrica. As características geométricas, físicas e mecânicas dos blocos protótipos foram determinadas em conformidade com a norma NBR 12.118 (ABNT, 2008).

A determinação da resistência e da condutividade térmica do concreto convencional e dos concretos contendo resíduo de Pinus spp. foi feita através de técnicas fluxométricas, utilizando um Condutivímetro Fluxométrico, conforme normas NBR 15.220-4 (ABNT, 2005) e ASTM C177 (2008).

A análise estatística dos resultados obtidos nos ensaios foi feita através da análise de variância (ANOVA) e, todas as vezes que a hipótese da nulidade foi rejeitada, efetuou-se a comparação das médias através do teste de Tukey-Kramer.

\section{RESULTADOS E DISCUSSÃO}

Nas Tabelas 3, 4 e 5 são apresentadas as resistências à compressão aos sete dias de idade (RC 7d) e as características físicas (massa específica, índice de vazios e absorção de água), dos concretos para blocos, traço 1:10, com as substituições do agregado miúdo convencional pelo resíduo de Pinus spp. Em função do coeficiente de variação determinado, de 9,48\%, o número mínimo de repetições para cada ensaio, foi fixado em quatro corpos de prova.

Analisando os valores apresentados nas Tabelas 3, 4 e 5, pode-se constatar que a RC $7 \mathrm{~d}$ apresenta diferença significativa entre cada teor de subs-

TABELA 3: Substituição do agregado miúdo convencional pelo resíduo de Pinus spp. in natura no concreto para blocos traço 1:10 - características físicas e mecânicas.

TABLE 3: Substitution of conventional fine aggregate by residues of Pinus spp.in natura in the concrete for blocks 1:10 - physical and mechanical characteristics.

\begin{tabular}{ccccccccc}
\hline $\begin{array}{c}\text { SV } \\
\text { Pinus } / \text { Nat }\end{array}$ & $\begin{array}{c}\text { RC 7d } \\
(\mathrm{MPa})\end{array}$ & $\begin{array}{c}\mathrm{CV} \\
(\%)\end{array}$ & $\begin{array}{c}\mathrm{ME} \\
\left(\mathrm{kg} / \mathrm{m}^{3}\right)\end{array}$ & $\begin{array}{c}\mathrm{CV} \\
(\%)\end{array}$ & $\begin{array}{c}\text { IV } \\
(\%)\end{array}$ & $\begin{array}{c}\mathrm{CV} \\
(\%)\end{array}$ & $\begin{array}{c}\text { ABS } \\
(\%)\end{array}$ & $\begin{array}{c}\mathrm{CV} \\
(\%)\end{array}$ \\
\hline Ref $0,0 \%$ & $10,26 \mathrm{a}$ & 6,25 & & 3,01 & $14,09 \mathrm{f}$ & 6,69 & $6,09 \mathrm{f}$ & 7,14 \\
$20,0 \%$ & $6,60 \mathrm{~b}$ & 5,83 & & 0,87 & $15,82 \mathrm{e}$ & 3,19 & $7,48 \mathrm{e}$ & 3,48 \\
$40,0 \%$ & $4,60 \mathrm{c}$ & 6,48 & & 1,03 & $18,99 \mathrm{~d}$ & 4,32 & $9,34 \mathrm{~d}$ & 5,23 \\
$60,0 \%$ & $3,10 \mathrm{~d}$ & 6,40 & & 2,18 & $22,14 \mathrm{c}$ & 6,95 & $11,69 \mathrm{c}$ & 3,93 \\
$80,0 \%$ & $2,95 \mathrm{e}$ & 2,95 & & 1,09 & $25,04 \mathrm{~b}$ & 3,86 & $14,45 \mathrm{~b}$ & 4,69 \\
$100,0 \%$ & $1,49 \mathrm{f}$ & 6,25 & $1.648,78 \mathrm{f}$ & 1,99 & $28,95 \mathrm{a}$ & 6,52 & $17,55 \mathrm{a}$ & 4,93 \\
\hline
\end{tabular}

Letras diferentes denotam diferenças estatísticas entre as médias das colunas ao nível de $95 \%$ de confiança; Médias obtidas de 4 medições; $\mathrm{SV}=$ Substituição em volume; Nat $=$ in natura $; \mathrm{RC} 7 \mathrm{~d}=$ Resistência à compressão aos 7 dias de cura; $\mathrm{CV}=$ Coeficiente de Variação; $\mathrm{ME}=$ Massa específica; IV = Índice de vazios; $\mathrm{ABS}=$ Absorção de água. 
TABELA 4: Substituição do agregado miúdo convencional pelo resíduo de Pinus spp. in natura com adição de 4,5\% de $\mathrm{CaCl}_{2} \cdot 2 \mathrm{H}_{2} \mathrm{O}$ no concreto para blocos traço 1:10 - características físicas e mecânicas.

TABLE 4: Substitution of conventional fine aggregate by residues of Pinus spp.in nature with addition of $4,5 \%$ of $\mathrm{CaCl}_{2} \cdot 2 \mathrm{H}_{2} \mathrm{O}$ in the concrete for blocks $1: 10$ - physical and mechanical characteristics.

\begin{tabular}{ccccccccc}
\hline $\begin{array}{c}\text { SV } \\
\text { Pinus } / \text { Nat/CC }\end{array}$ & $\begin{array}{c}\text { RC 7d } \\
(\mathrm{MPa})\end{array}$ & $\begin{array}{c}\mathrm{CV} \\
(\%)\end{array}$ & $\begin{array}{c}\mathrm{ME} \\
\left(\mathrm{kg} / \mathrm{m}^{3}\right)\end{array}$ & $\begin{array}{c}\mathrm{CV} \\
(\%)\end{array}$ & $\begin{array}{c}\text { IV } \\
(\%)\end{array}$ & $\begin{array}{c}\mathrm{CV} \\
(\%)\end{array}$ & $\begin{array}{c}\text { ABS } \\
(\%)\end{array}$ & $\begin{array}{c}\mathrm{CV} \\
(\%)\end{array}$ \\
\hline Ref $0,0 \%$ & $10,26 \mathrm{a}$ & 6,25 & $2.313,09 \mathrm{a}$ & 3,01 & $14,09 \mathrm{f}$ & 6,69 & $6,09 \mathrm{f}$ & 7,14 \\
$20,0 \%$ & $7,48 \mathrm{~b}$ & 6,25 & $2.022,81 \mathrm{~b}$ & 1,77 & $16,48 \mathrm{e}$ & 5,01 & $8,15 \mathrm{e}$ & 5,61 \\
$40,0 \%$ & $5,11 \mathrm{c}$ & 4,25 & $1.947,30 \mathrm{c}$ & 0,87 & $19,60 \mathrm{~d}$ & 4,49 & $10,07 \mathrm{~d}$ & 5,22 \\
$60,0 \%$ & $3,68 \mathrm{~d}$ & 6,36 & $1.827,99 \mathrm{~d}$ & 0,69 & $21,78 \mathrm{c}$ & 1,09 & $11,91 \mathrm{c}$ & 0,82 \\
$80,0 \%$ & $2,69 \mathrm{e}$ & 4,54 & $1.699,44 \mathrm{e}$ & 1,31 & $26,55 \mathrm{~b}$ & 3,62 & $15,63 \mathrm{~b}$ & 4,86 \\
$100,0 \%$ & $1,73 \mathrm{f}$ & 7,06 & $1.633,64 \mathrm{f}$ & 3,21 & $27,93 \mathrm{a}$ & 4,51 & $17,12 \mathrm{a}$ & 6,86 \\
\hline
\end{tabular}

Letras diferentes denotam diferenças estatísticas entre as médias das colunas ao nível de $95 \%$ de confiança; Médias obtidas de 4 medições; $\mathrm{SV}=$ Substituição em volume; $\mathrm{Nat}=$ in natura $; \mathrm{CC}=\mathrm{CaCl}_{2} \cdot 2 \mathrm{H}_{2} \mathrm{O} ; \mathrm{RC} 7 \mathrm{~d}=$ Resistência à compressão aos 7 dias de cura; $\mathrm{CV}=$ Coeficiente de Variação; $\mathrm{ME}=$ Massa específica; IV = Índice de vazios; $\mathrm{ABS}$ = Absorção de água.

TABELA 5: Substituição do agregado miúdo convencional pelo resíduo de Pinus spp. AF48H com adição de $4,5 \%$ de $\mathrm{CaCl}_{2} \cdot 2 \mathrm{H}_{2} \mathrm{O}$ no concreto para blocos traço 1:10 - características físicas e mecânicas.

TABLE 5: Substitution of conventional fine aggregate by residues of Pinus spp.AF-48H with addition of $4,5 \%$ of $\mathrm{CaCl}_{2} .2 \mathrm{H}_{2} \mathrm{O}$ in concrete for blocks 1:10 - physical and mechanical characteristics.

\begin{tabular}{ccccccccc}
\hline $\begin{array}{c}\text { SV } \\
\text { Pinus } / A F 48 H / C C\end{array}$ & $\begin{array}{c}\text { RC 7d } \\
(\mathrm{MPa})\end{array}$ & $\begin{array}{c}\mathrm{CV} \\
(\%)\end{array}$ & $\begin{array}{c}\mathrm{ME} \\
\left(\mathrm{kg} / \mathrm{m}^{3}\right)\end{array}$ & $\begin{array}{c}\mathrm{CV} \\
(\%)\end{array}$ & $\begin{array}{c}\mathrm{IV} \\
(\%)\end{array}$ & $\begin{array}{c}\mathrm{CV} \\
(\%)\end{array}$ & $\begin{array}{c}\text { ABS } \\
(\%)\end{array}$ & $\begin{array}{c}\mathrm{CV} \\
(\%)\end{array}$ \\
\hline Ref $0,0 \%$ & $10,26 \mathrm{a}$ & 6,25 & $2.313,09 \mathrm{a}$ & 3,01 & $14,09 \mathrm{f}$ & 6,69 & $6,09 \mathrm{f}$ & 7,14 \\
$20,0 \%$ & $8,37 \mathrm{~b}$ & 3,38 & $2.085,76 \mathrm{~b}$ & 3,04 & $15,91 \mathrm{e}$ & 4,82 & $7,54 \mathrm{e}$ & 5,33 \\
$40,0 \%$ & $6,00 \mathrm{c}$ & 5,77 & $1.991,72 \mathrm{c}$ & 3,75 & $18,59 \mathrm{~d}$ & 7,19 & $9,36 \mathrm{~d}$ & 6,33 \\
$60,0 \%$ & $3,86 \mathrm{~d}$ & 4,79 & $1.843,43 \mathrm{~d}$ & 1,85 & $23,13 \mathrm{c}$ & 7,88 & $12,57 \mathrm{c}$ & 9,99 \\
$80,0 \%$ & $3,24 \mathrm{e}$ & 5,41 & $1.700,97 \mathrm{e}$ & 3,04 & $25,48 \mathrm{~b}$ & 7,50 & $15,01 \mathrm{~b}$ & 8,21 \\
$100,0 \%$ & $1,74 \mathrm{f}$ & 5,90 & $1.649,49 \mathrm{f}$ & 3,28 & $28,81 \mathrm{a}$ & 2,44 & $17,48 \mathrm{a}$ & 2,93 \\
\hline
\end{tabular}

Letras diferentes denotam diferenças estatísticas entre as médias das colunas ao nível de $95 \%$ de confiança; Médias obtidas de 4 medições; $\mathrm{SV}=$ Substituição em volume; $\mathrm{AF} 48 \mathrm{H}$ = Pré-tratamento tipo extração em água fria por 48 horas; $\mathrm{CC}=\mathrm{CaCl}_{2} \cdot 2 \mathrm{H}_{2} \mathrm{O} ; \mathrm{RC} 7 \mathrm{~d}=$ Resistência à compressão aos 7 dias de cura; $\mathrm{CV}=$ Coeficiente de Variação; $\mathrm{ME}=$ Massa específica; IV = Índice de vazios; ABS = Absorção de água.

tituição do agregado miúdo mineral pelo resíduo de Pinus spp. (in natura, in natura com adição de 4,5\% de $\mathrm{CaCl}_{2} .2 \mathrm{H}_{2} \mathrm{O}$ e com o pré-tratamento $\mathrm{AF} 48 \mathrm{H}$ e com adição de $4,5 \%$ de $\mathrm{CaCl}_{2} \cdot 2 \mathrm{H}_{2} \mathrm{O}$ ), sendo que a queda de resistência, para teores de substituição de $100 \%$ do agregado miúdo mineral, chega a $85,48 \%$ para o resíduo de Pinus spp. in natura, $83,14 \%$ para o resíduo de Pinus spp. in natura com adição de $4,5 \%$ de $\mathrm{CaCl}_{2} \cdot 2 \mathrm{H}_{2} \mathrm{O}$, e $83,04 \%$ para o resíduo com pré-tratamento $\mathrm{AF} 48 \mathrm{H}$ e $4,5 \%$ de $\mathrm{CaCl}_{2} \cdot 2 \mathrm{H}_{2} \mathrm{O}$.

A massa específica do concreto também apresenta diferença estatística significativa para teor de substituição analisado, para qualquer um dos três tipos de resíduo de Pinus spp. utilizados, sendo que a maior diferença determinada foi de $29,37 \%$ de redução (100\% de substituição por resíduo de Pinus spp. in natura com adição de $4,5 \%$ de $\mathrm{CaCl}_{2} \cdot 2 \mathrm{H}_{2} \mathrm{O}$ ).

A porosidade do material, expressa pelo seu índice de vazios, também apresentou diferença significativa entre todas as proporções de agregado miúdo mineral e resíduo de Pinus spp., sendo que o maior valor determinado foi para $100 \%$ de substituição de Pinus spp. in natura (aumento de 105,46\%).

A absorção de água, como esperado, também apresentou diferença significativa para todos os teores de substituição pelos três tipos de serragem 
de Pinus spp. testados, sendo que o maior incremento de absorção de água foi verificado para $100 \%$ de substituição de Pinus spp. in natura (188,18\%).

Fica evidenciada a melhora da compatibilidade entre o resíduo de Pinus spp. e o cimento Portland, em função da adição de $4,5 \%$ de $\mathrm{CaCl}_{2} \cdot 2 \mathrm{H}_{2} \mathrm{O}$ ao compósito, conforme indicado por Simatupang et al. (1988) e Semple e Evans (2004), traduzida pelo aumento médio de $10,08 \%$ da resistência à compressão aos sete dias de idade ao concreto produzido com resíduo de Pinus spp. in natura. A utilização do pré-tratamento AF48H, conforme Simatupang et al. (1988), também conferiu melhoras significativas nas resistências à compressão do concreto com resíduo de Pinus spp., pois apresentou um ganho médio de $11,05 \%$ em compa- ração com o material produzido com $\mathrm{CaCl}_{2} \cdot \mathrm{H}_{2} \mathrm{O}$ e sem o pré-tratamento e de $21,68 \%$ em comparação ao concreto produzido com o resíduo de Pinus spp. in natura e sem o acelerador de pega.

Quanto às características físicas determinadas, não se verificaram variações significativas em função do uso de $4,5 \%$ de $\mathrm{CaCl}_{2} \cdot \mathrm{H}_{2} \mathrm{O}$ ou do uso concomitante do pré-tratamento tipo $\mathrm{AF} 48 \mathrm{H}$, mais $4,5 \%$ de $\mathrm{CaCl}_{2} \cdot \mathrm{H}_{2} \mathrm{O}$.

$\mathrm{Na}$ Figura 1, são ilustradas as dispersões dos valores da resistência à compressão aos sete dias de idade e das características físicas dos concretos com substituições do agregado miúdo convencional pelo resíduo de Pinus spp., bem como os modelos que proporcionam o melhor ajuste destas características em função dos teores de substituição.
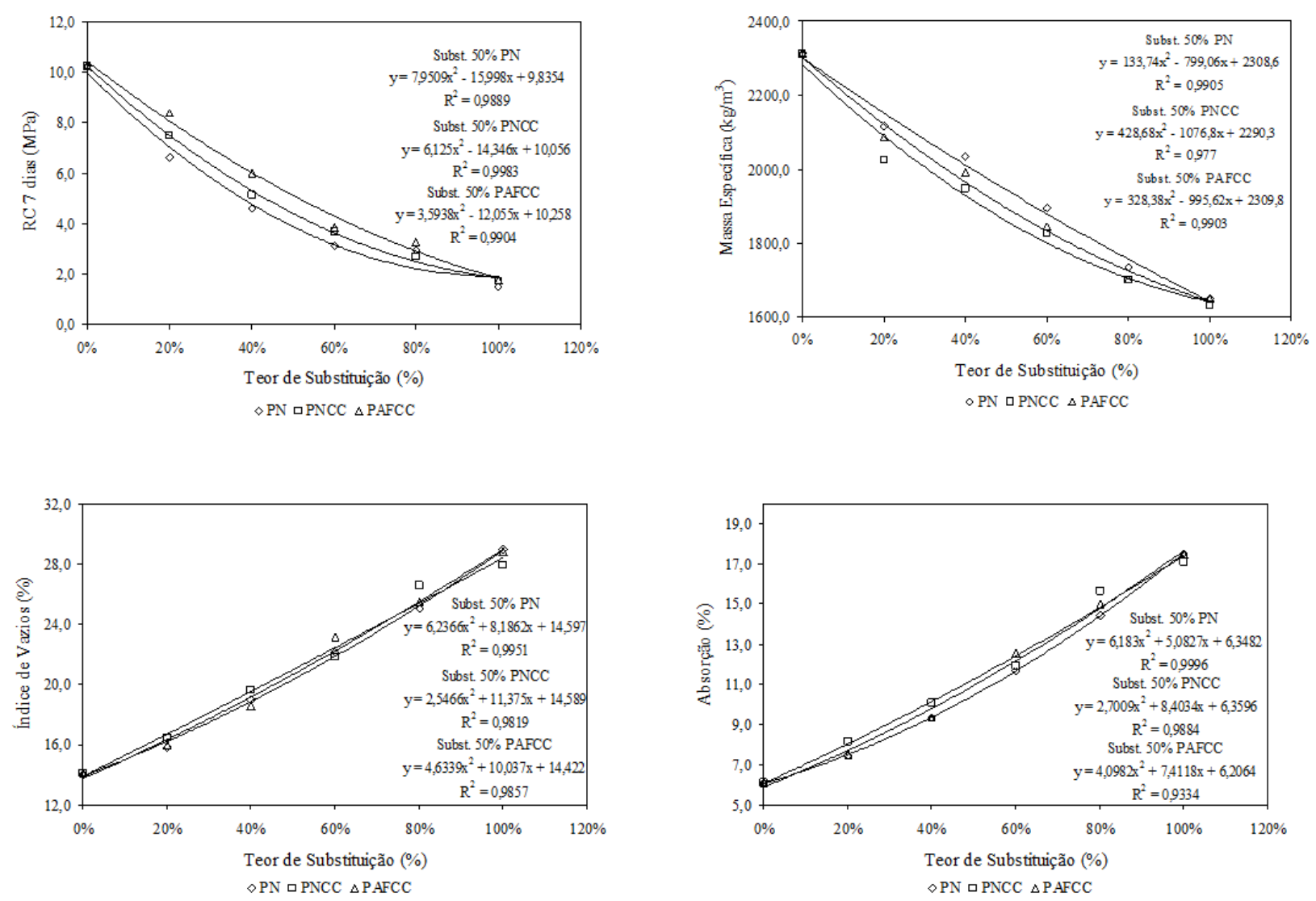

FIGURA 1: Características do concreto com substituição do agregado miúdo convencional pelo resíduo de Pinus spp. in natura (PN), Pinus spp. in natura e com adição de $4,5 \%$ de $\mathrm{CaCl}_{2} \cdot 2 \mathrm{H}_{2} \mathrm{O}$ (PNCC) e Pinus spp. com extração em água fria por $48 \mathrm{~h}$ com adição de $4,5 \%$ de $\mathrm{CaCl}_{2} \cdot 2 \mathrm{H}_{2} \mathrm{O}$ (APAFCC).

FIGURE 1: Characteristics of concrete with substitution of conventional fine aggregate by residue of Pinus spp. in nature (PN), Pinus spp. in nature with addition of $4,5 \%$ of $\mathrm{CaCl}_{2} \cdot 2 \mathrm{H}_{2} \mathrm{O}$ (PNCC) and Pinus spp. with extraction in cold water for 48 hours with addition of $4,5 \%$ of $\mathrm{CaCl}_{2} \cdot 2 \mathrm{H}_{2} \mathrm{O}$ (APAFCC). 
Em função da análise dos modelos de regressão apresentados na Figura 1 e objetivando-se obter um bloco de concreto com RC 7d acima da resistência mínima de 3,0 $\mathrm{MPa}$, estabelecida pela norma NBR 6.136 (ABNT, 2008) para uso em alvenaria estrutural, optou-se por uma substituição do agregado miúdo convencional por $50 \%$ de resíduo de Pinus spp., o que resultou nas expectativas de valores para resistência aos sete dias de cura (RC 7d exp) e características físicas, apresentadas na Tabela 6.

Para confirmar os valores previstos pelos modelos de regressão, apresentados na Tabela 6, foram produzidas três séries de corpos de prova cilíndricos de $\mathrm{Dn}=50 \mathrm{~mm}$, uma para cada tipo de resíduo de Pinus spp. As séries foram, então, ensaiadas para determinação das características físicas e mecânicas e seus resultados comparados com o traço de referência (concreto para blocos traço 1:10, com $100 \%$ de agregado miúdo mineral). Os valores assim determinados são apresentados na Tabela 7.

Analisando os valores apresentados na Tabela 7, constata-se que tanto a resistência à compressão quanto as características mecânicas, determinadas com o ensaio dos corpos de prova, se aproximaram muito dos valores previstos pelas equações de regressão apresentadas na Figura 1.

Em relação ao concreto de referência, as massas específicas dos concretos contendo resíduo de Pinus spp., apresentaram diferenças significativas, com valores em média $15,40 \%$ menores.

Quanto à porosidade do concreto, verifica-se que os valores do índice de vazios do concreto com resíduo de Pinus spp., apresentaram aumentos significativos quando comparados com o con- creto com $100 \%$ de agregado mineral, da ordem de $37,33 \%$ (substituição por $50 \%$ de Pinus spp. in natura), 44,10\% (substituição por $50 \%$ de serragem de Pinus spp. in natura com $4,5 \%$ de $\mathrm{CaCl}_{2} \cdot 2 \mathrm{H}_{2} \mathrm{O}$ ) e $47,59 \%$ (substituição por $50 \%$ de resíduo de Pinus spp. AF48H com adição de $4,5 \%$ de $\left.\mathrm{CaCl}_{2} \cdot 2 \mathrm{H}_{2} \mathrm{O}\right)$.

Com relação à absorção de água, os concretos com resíduo de Pinus spp. apresentaram aumentos significativos quando comparados com o concreto com $100 \%$ de agregado mineral, da ordem

TABELA 6: Concreto para blocos traço 1:10 com substituição de $50 \%$ do agregado miúdo convencional por resíduo de Pinus spp. - expectativa de valores para resistência e características físicas.

TABLE 6: Concrete for blocks 1:10 with substitution of $50 \%$ of conventional fine aggregate by residue of Pinus spp.- expectation of values for resistance and physical characteristics.

\begin{tabular}{ccccc}
\hline S.Ag.Mi & $\begin{array}{c}\text { RC 7d exp } \\
(\mathrm{MPa})\end{array}$ & $\begin{array}{c}\mathrm{ME}_{\text {exp }} \\
\left(\mathrm{kg} / \mathrm{m}^{3}\right)\end{array}$ & $\begin{array}{c}\mathrm{IV}_{\text {exp }} \\
(\%)\end{array}$ & $\begin{array}{c}\mathrm{ABS}_{\text {exp }} \\
(\%)\end{array}$ \\
\hline $50 \mathrm{PN}$ & 3,82 & $1.942,51$ & 20,25 & 10,44 \\
$50 \mathrm{PNCC}$ & 4,41 & $1.859,07$ & 20,91 & 11,24 \\
$50 \mathrm{PAFCC}$ & 5,13 & $1.894,09$ & 20,60 & 10,94 \\
\hline
\end{tabular}

S.Ag.Mi $=$ Substituição do agregado miúdo; $50 \mathrm{PN}=$ $50 \%$ Pinus spp. in natura; 50PNCC $=50 \%$ Pinus in natura $+4,5 \%$ de $\mathrm{CaCl}_{2} \cdot 2 \mathrm{H}_{2} \mathrm{O} ; 50 \mathrm{PAFCC}=50 \%$ Pinus spp. com pré-tratamento em água fria $+4,5 \% \mathrm{de} \mathrm{CaCl}_{2} \cdot 2 \mathrm{H}_{2} \mathrm{O}$; $\mathrm{RC} 7 \mathrm{~d}$ exp $=$ Expectativa de resistência à compressão aos 7 dias de cura; ME exp = Expectativa de massa específica; IV exp = Expectativa de índice de vazios; ABS exp = Expectativa de absorção de água.

TABELA 7: Concreto para blocos traço 1:10 com substituição de 50\% do agregado miúdo convencional por resíduo de Pinus spp. - características físicas.

TABLE 7: Concrete for blocks 1:10 with substitution of 50\% of conventional fine aggregate by residue of Pinus spp.- physical characteristics.

\begin{tabular}{ccccccccc}
\hline S.Ag.Mi & $\begin{array}{c}\mathrm{RC} 7 \mathrm{~d} \\
(\mathrm{MPa})\end{array}$ & $\begin{array}{c}\mathrm{CV} \\
(\%)\end{array}$ & $\begin{array}{c}\mathrm{ME} \\
\left(\mathrm{kg} / \mathrm{m}^{3}\right)\end{array}$ & $\begin{array}{c}\mathrm{CV} \\
(\%)\end{array}$ & $\begin{array}{c}\text { IV } \\
(\%)\end{array}$ & $\begin{array}{c}\mathrm{CV} \\
(\%)\end{array}$ & $\begin{array}{c}\text { ABS } \\
(\%)\end{array}$ & $\begin{array}{c}\mathrm{CV} \\
(\%)\end{array}$ \\
\hline Ref $1: 10$ & $10,26 \mathrm{a}$ & 2,50 & & 2,50 & $14,92 \mathrm{~d}$ & 3,98 & $6,43 \mathrm{~d}$ & 6,15 \\
50PN & $4,18 \mathrm{c}$ & 0,80 & & 0,80 & $20,49 \mathrm{~b}$ & 4,58 & $10,44 \mathrm{~b}$ & 5,03 \\
50PNCC & $4,66 \mathrm{bc}$ & 0,64 & & 0,64 & $17,50 \mathrm{c}$ & 1,58 & $8,78 \mathrm{c}$ & 2,13 \\
50PAFCC & $5,13 \mathrm{~b}$ & 2,22 & & 2,22 & $22,02 \mathrm{a}$ & 4,64 & $11,36 \mathrm{a}$ & 5,70 \\
\hline
\end{tabular}

Letras diferentes denotam diferenças estatísticas entre as médias das colunas ao nível de $95 \%$ de confiança; Médias obtidas de 4 medições; S.Ag.Mi = Substituição do agregado miúdo; Ref 1:10 = Concreto de referência Traço 1:10; $50 \mathrm{PN}=50 \%$ Pinus spp. in natura; $50 \mathrm{PNCC}=50 \%$ Pinus spp. in natura $+4,5 \% \mathrm{de} \mathrm{CaCl}_{2} \cdot 2 \mathrm{H}_{2} \mathrm{O} ; 50 \mathrm{PAFCC}=50 \%$ Pinus spp. com pré-tratamento em água fria $+4,5 \% \mathrm{de} \mathrm{CaCl}_{2} .2 \mathrm{H}_{2} \mathrm{O} ; \mathrm{RC} 7 \mathrm{~d}=$ Resistência à compressão aos 7 dias de cura; $\mathrm{CV}=$ Coeficiente de Variação; $\mathrm{ME}=$ Massa específica; IV = Índice de vazios; $\mathrm{ABS}=$ Absorção de água. 
de $62,36 \%$ (substituição por $50 \%$ de Pinus spp. in natura), $83,20 \%$ (substituição por $50 \%$ de resíduo de Pinus spp. in natura com $4,5 \%$ de $\mathrm{CaCl}_{2} .2 \mathrm{H}_{2} \mathrm{O}$ ) e $76,67 \%$ (substituição por $50 \%$ de resíduo de Pinus spp. AF48H com adição de 4,5\% de $\mathrm{CaCl}_{2} \cdot 2 \mathrm{H}_{2} \mathrm{O}$ ).

Quanto à RC 7d, constata-se que as diferenças apresentadas em relação ao traço com materiais convencionais, apresentaram a seguinte variação: redução de $59,26 \%(50 \%$ de resíduo de Pinus spp. in natura), redução de 54,58\% (50\% de resíduo de Pinus spp. in natura com adição de 4,5\% de $\left.\mathrm{CaCl}_{2} \cdot 2 \mathrm{H}_{2} \mathrm{O}\right)$ e redução de $50,00 \%$ (50\% de resíduo de Pinus spp. AF48H com adição de $4,5 \%$ de $\mathrm{CaCl}_{2} \cdot 2 \mathrm{H}_{2} \mathrm{O}$ ).

Os resultados obtidos validaram a produção dos blocos com substituição de $50 \%$ do volume do agregado miúdo convencional pelos resíduos de Pinus spp.

Durante a produção dos blocos com $50 \%$ do agregado miúdo convencional substituído pelo resíduo de Pinus spp., verificou-se que os blocos invariavelmente apresentavam trincas (Figura 2) o que poderia inviabilizar a produção. Diante deste problema, resolveu-se diminuir a velocidade de desforma (abertura dos extratores). $\mathrm{O}$ aumento de dois segundos para cinco segundos se mostrou suficiente para a produção de blocos sem defeitos aparentes.

Os valores determinados para as características físicas dos blocos são apresentados na Tabela 8. As características mecânicas, no caso as resistências à compressão aos três dias (RC 3d), sete dias (RC 7d) e 28 dias (RC 28d), são apresentadas na Tabela 9.

Como pode ser verificado na Tabela 8 , tanto a massa específica quanto a porosidade e a absorção de água dos blocos produzidos com 50\% de resíduo de Pinus spp. apresentaram diferenças significativas a $95 \%$ de confiança em relação aos blocos convencionais de concreto. A retração por secagem, por sua vez, não apresentou diferenças entre os blocos protótipos, nem entre estes e o bloco de concreto convencional, de referência, confirmando a excelente estabilidade dimensional dos compósitos cimento-madeira, conforme relatado por Jorge et al. (2004).

Quanto aos valores apresentados, verifica-se uma redução de 10,32\% (média) da massa dos
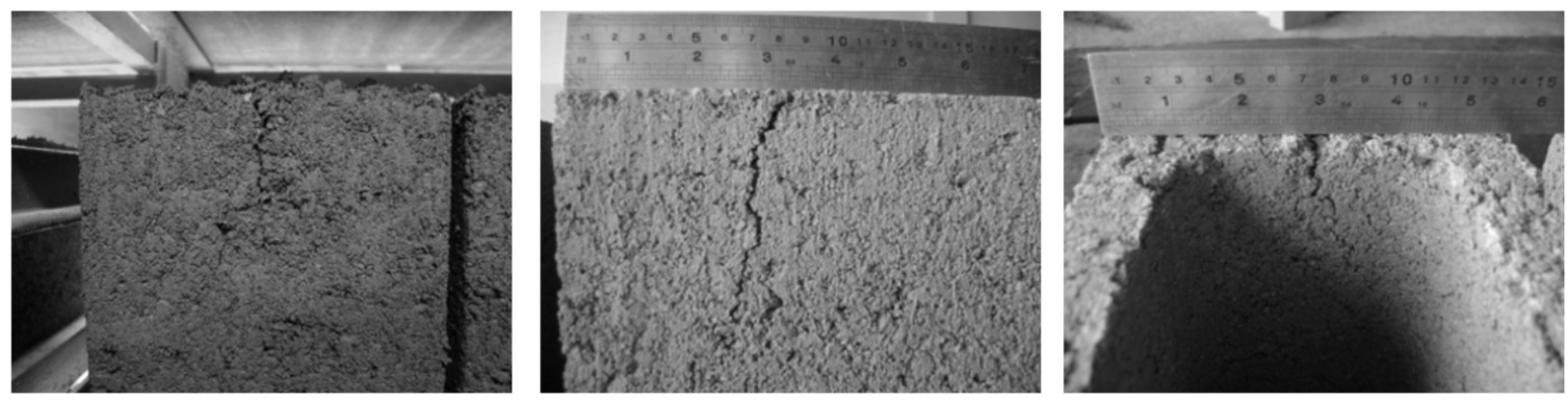

FIGURA 2: Exemplos de trincas em blocos devido à velocidade de desforma.

FIGURE 2: Examples of split in blocks due to defaced speed.

TABELA 8: Características físicas dos blocos protótipos.

TABLE 8: Physical characteristics of prototype blocks.

\begin{tabular}{ccccccccc}
\hline S.Ag.Mi & $\begin{array}{c}\text { ME } \\
\left(\mathrm{kg} / \mathrm{m}^{3}\right)\end{array}$ & $\begin{array}{c}\text { CV } \\
(\%)\end{array}$ & $\begin{array}{c}\text { IV } \\
(\%)\end{array}$ & $\begin{array}{c}\text { CV } \\
(\%)\end{array}$ & $\begin{array}{c}\text { ABS } \\
(\%)\end{array}$ & $\begin{array}{c}\text { CV } \\
(\%)\end{array}$ & $\begin{array}{c}\text { RS } \\
(\%)\end{array}$ & $\begin{array}{c}\text { CV } \\
(\%)\end{array}$ \\
\hline Ref $1: 10$ & $2.242,36 \mathrm{a}$ & 0,85 & $12,12 \mathrm{~d}$ & 1,88 & $5,40 \mathrm{~d}$ & 2,71 & $0,0446 \mathrm{a}$ & 13,99 \\
50PN & $2.019,38 \mathrm{~b}$ & 1,95 & $14,54 \mathrm{~b}$ & 5,49 & $7,21 \mathrm{~b}$ & 7,36 & $0,0422 \mathrm{a}$ & 4,06 \\
50PNCC & $1.977,97 \mathrm{c}$ & 1,42 & $15,51 \mathrm{a}$ & 3,19 & $7,84 \mathrm{a}$ & 2,21 & $0,0446 \mathrm{a}$ & 9,14 \\
50PAFCC & $2.035,34 \mathrm{~b}$ & 0,72 & $13,47 \mathrm{c}$ & 1,36 & $6,62 \mathrm{c}$ & 1,76 & $0,0443 \mathrm{a}$ & 5,43 \\
\hline
\end{tabular}

Letras diferentes denotam diferenças estatísticas entre as médias das colunas ao nível de $95 \%$ de confiança; Médias obtidas de 4 medições; S.Ag.Mi = Substituição do agregado miúdo; Ref 1:10 = Concreto de referência Traço 1:10; $50 \mathrm{PN}=50 \%$ Pinus spp. in natura; $50 \mathrm{PNCC}=50 \%$ Pinus spp. in natura $+4,5 \% \mathrm{de} \mathrm{CaCl}_{2} \cdot 2 \mathrm{H}_{2} \mathrm{O} ; 50 \mathrm{PAFCC}=50 \%$ Pinus spp. com pré-tratamento em água fria $+4,5 \%$ de $\mathrm{CaCl}_{2} \cdot 2 \mathrm{H}_{2} \mathrm{O} ; \mathrm{ME}=$ Massa específica; $\mathrm{CV}=$ Coeficiente de Variação IV = Índice de vazios; ABS = Absorção de água. 
TABELA 9: Características mecânicas dos blocos protótipos.

TABLE 9: Mechanical characteristics of prototype blocks.

\begin{tabular}{ccccccc}
\hline Blocos protótipos & \multicolumn{7}{c}{ Resistência à compressão (MPa) } \\
\cline { 2 - 7 } S.Ag.Mi & RC 3d & CV (\%) & RC 7d & CV (\%) & RC 28d & CV (\%) \\
\hline Ref 1:10 & $5,99 \mathrm{a}$ & 6,98 & $10,83 \mathrm{a}$ & 2,60 & $13,06 \mathrm{a}$ & 3,86 \\
50PN & $2,64 \mathrm{c}$ & 6,48 & $3,80 \mathrm{c}$ & 7,20 & $4,39 \mathrm{c}$ & 8,68 \\
50PNCC & $3,35 \mathrm{~b}$ & 7,08 & $4,21 \mathrm{~b}$ & 6,07 & $4,61 \mathrm{bc}$ & 3,58 \\
50PAFCC & $3,48 \mathrm{~b}$ & 4,27 & $4,57 \mathrm{~b}$ & 4,53 & $5,16 \mathrm{~b}$ & 7,19 \\
\hline
\end{tabular}

Letras diferentes denotam diferenças estatísticas entre as médias das colunas ao nível de 95\% de confiança; Médias obtidas de 4 medições; S.Ag.Mi = Substituição do agregado miúdo; Ref 1:10 = Concreto de referência Traço 1:10; $50 \mathrm{PN}=50 \%$ Pinus spp. in natura; $50 \mathrm{PNCC}=50 \%$ Pinus spp. in natura $+4,5 \%$ de $\mathrm{CaCl}_{2} \cdot 2 \mathrm{H}_{2} \mathrm{O} ; 50 \mathrm{PAFCC}=50 \%$ Pinus spp. com pré-tratamento em água fria $+4,5 \%$ de $\mathrm{CaCl}_{2} \cdot 2 \mathrm{H}_{2} \mathrm{O} ; \mathrm{RC} 3 \mathrm{~d}=$ Resistência à compressão aos 3 dias de cura; RC 7d = Resistência à compressão aos 7 dias de cura; RC 28d = Resistência à compressão aos 28 dias de cura; $\mathrm{CV}=$ Coeficiente de Variação.

blocos em função da substituição parcial do agregado graúdo convencional pelos resíduos de Pinus spp.

Os valores de absorção de água, apresentados pelos blocos protótipos, apesar de maiores que o valor médio determinado para o bloco de concreto convencional, são menores que os limites estabelecidos pela norma brasileira ABNT NBR 6.136 (ABNT, 2008), com valores máximos de $10 \%$ para blocos produzidos com agregados minerais convencionais e $13 \%$ para blocos produzidos com agregados leves.

Os valores de retração por secagem, determinados para os blocos protótipos, também se apresentam menores que o limite de $0,065 \%$ estabelecido pela NBR 6.136 (ABNT, 2008).

Quanto às resistências à compressão, apresentadas na Tabela 9, os blocos protótipos, com substituição de $50 \%$ do agregado miúdo convencional apresentaram, para todas as idades, valores significativamente inferiores aos apresentados pelos blocos convencionais. Em comparação aos blocos convencionais de referência, os valores médios de resistências dos blocos protótipos apresentaram reduções médias de 47,25\% aos 3 dias, $61,31 \%$ aos 7 dias e $63,86 \%$ aos 28 dias. Porém, aos sete dias de idade, todos os blocos protótipos apresentaram resistências à compressão acima do limite fixado pela NBR 6.136 (ABNT, 2008) para utilização em alvenarias estruturais, que é de 3,0 MPa.

As resistências à compressão aos sete dias determinadas para os blocos protótipos apresentaram reduções médias de $9,89 \%$ em relação às resistências determinadas com os corpos de prova cilíndricos moldados em laboratório. Tal redução se verificou em virtude das condições diferenciadas de produção e cura do material.

A evolução das resistências à compressão com a idade dos blocos de referência e protótipos pode ser verificada na Figura 3.

$\mathrm{Na}$ Figura 3 ficam evidenciadas as diferenças significativas das resistências à compressão em todas as idades, quando comparadas às resistências determinadas para os blocos produzidos com o concreto convencional. Porém, apesar dessas reduções, os valores das resistências à compressão determinadas com os blocos protótipos, contendo substituição de $50 \%$ em volume do agregado miúdo convencional (areia) pelo resíduo de Pinus spp. apresentaram valores acima da mínima para uso em alvenaria estrutural, que, conforme a normalização brasileira, é de 3,0 MPa.

Os resultados de condutividade térmica dos materiais com os quais os blocos protótipos foram produzidos são apresentados na Tabela 10.

Como pode ser verificado, a substituição de $50 \%$ em volume do agregado miúdo convencional pelo resíduo de Pinus spp. fez com que a condutividade térmica média do concreto para blocos traço $1: 10$, fosse reduzida de $0,90 \mathrm{~W} / \mathrm{mK}$, para valores em torno de $0,60 \mathrm{~W} / \mathrm{mK}$ (resíduo de Pinus spp. AF48H $+4,5 \%$ de $\mathrm{CaCl}_{2} \cdot 2 \mathrm{H}_{2} \mathrm{O}$ ) a $0,64 \mathrm{~W} / \mathrm{mK}$ (resíduo de Pinus spp. in natura $+4,5 \%$ de $\mathrm{CaCl}_{2} \cdot 2 \mathrm{H}_{2} \mathrm{O}$ ). Portanto, com reduções estatisticamente significativas da ordem de $33,33 \%$ a $28,89 \%$, o que confirma a tese de Savastano Júnior e Agopyan (1997) de que os materiais produzidos com base em compósitos cimento-madeira apresentam características de isolamento térmico, superiores aos materiais convencionais de construção, tais como os blocos 


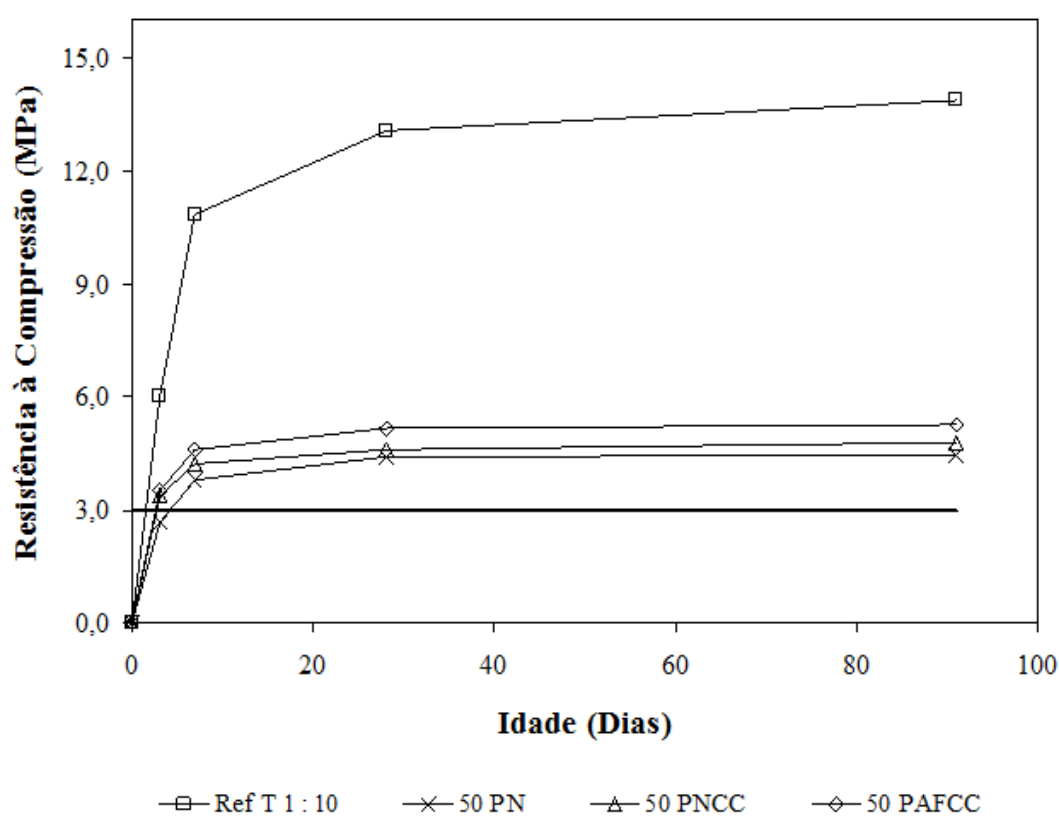

FIGURA 3: Evolução da resistência à compressão com a idade: Ref T 1:10 - blocos de referência de concreto convencional Traço 1:10, 50PN - blocos com substituição de 50\% do agregado miúdo mineral por Pinus spp. in natura, 50PNCC - blocos com substituição de $50 \%$ do agregado miúdo mineral por Pinus spp. in natura $+4,5 \%$ de $\mathrm{CaCl}_{2} \cdot 2 \mathrm{H}_{2} \mathrm{O}, 50 \mathrm{PAFCC}$ - blocos com substituição de $50 \%$ do agregado miúdo mineral por Pinus spp. com extração em água fria por $48 \mathrm{~h}+4,5 \% \mathrm{de} \mathrm{CaCl}_{2} \cdot 2 \mathrm{H}_{2} \mathrm{O}$.

FIGURE 3: Evolution of compression strength with ages. Ref T 1:10 - referential blocks of conventional concrete $\mathrm{T} 1: 10,50 \mathrm{PN}$ - blocks with $50 \%$ substitution of conventional fine aggregate by residue of Pinus spp. in nature, 50PNCC - blocks with 50\% substitution of conventional fine aggregate by residue of Pinus spp. in nature with addition of $4,5 \%$ of $\mathrm{CaCl}_{2} \cdot 2 \mathrm{H}_{2} \mathrm{O}, 50 \mathrm{PAFCC}$ - blocks with 50\% substitution of conventional fine aggregate by residue of and Pinus spp. with extraction in cold water for 48 hours with addition of $4,5 \%$ of $\mathrm{CaCl}_{2} \cdot 2 \mathrm{H}_{2} \mathrm{O}$.

TABELA 10:Condutividade térmica do concreto dos blocos.

TABLE 10: Thermal conductivity of concrete of blocks.

\begin{tabular}{cccc}
\hline $\begin{array}{c}\text { Concreto } \\
\text { S.Ag.Mi }\end{array}$ & $\begin{array}{c}\text { CT }(\mathrm{W} / \\
\mathrm{mK})\end{array}$ & $\mathrm{CV}(\%)$ & $\begin{array}{c}\text { Variação } \\
(\%)\end{array}$ \\
\hline Ref $1: 10$ & $0,90 \mathrm{a}$ & 0,64 & - \\
50PN & $0,61 \mathrm{~b}$ & 9,13 & $-32,22$ \\
50PNCC & $0,64 \mathrm{c}$ & 2,40 & $-28,89$ \\
50PAFCC & $0,60 \mathrm{~d}$ & 3,83 & $-33,33$ \\
\hline
\end{tabular}

Letras diferentes denotam diferenças estatísticas entre as médias das colunas ao nível de $95 \%$ de confiança; Médias obtidas de 4 medições; S.Ag.Mi = Substituição do agregado miúdo; Ref 1:10 = Concreto de referência Traço $1: 10 ; 50 \mathrm{PN}=50 \%$ Pinus spp. in natura; $50 \mathrm{PNCC}=50 \%$ Pinus spp.in natura $+4,5 \%$ de $\mathrm{CaCl}_{2} \cdot 2 \mathrm{H}_{2} \mathrm{O} ; 50 \mathrm{PAFCC}$ $=50 \%$ Pinus spp. com pré-tratamento em água fria + $4,5 \%$ de $\mathrm{CaCl}_{2} .2 \mathrm{H}_{2} \mathrm{O}$; $\mathrm{CT}=$ Condutividade térmica; $\mathrm{CV}$ $=$ Coeficiente de Variação. cerâmicos, que conforme a NBR 15.220-4 (ABNT, 2005), possuem condutividade térmica variando entre $0,70 \mathrm{~W} / \mathrm{mK}$ a $1,05 \mathrm{~W} / \mathrm{mK}$.

\section{CONCLUSÕES}

Com base nos resultados obtidos nesta pesquisa, as seguintes conclusões podem ser apresentadas:

A utilização do resíduo de Pinus spp. com diâmetro máximo de $4,8 \mathrm{~mm}$, como substituição parcial do agregado miúdo mineral para a produção de blocos de concreto para uso em alvenarias estruturais é tecnicamente viável;

A utilização de $\mathrm{CaCl}_{2} \cdot 2 \mathrm{H}_{2} \mathrm{O}$ como aditivo acelerador de pega propiciou ganhos significativos de resistência dos blocos com resíduo de Pinus spp.;

A utilização de pré-tratamento, tipo extração em água fria por 48 horas (AF48H), propiciou 
aumentos significativos da resistência à compressão dos blocos, quando comparados aos blocos produzidos com resíduo de Pinus spp. sem tratamento;

Verificou-se o aparecimento de fissuras na produção dos blocos com resíduo de Pinus spp., problema facilmente resolvido com pequena alteração no processo de fabricação;

Diante dos limites estabelecidos pela normalização brasileira, os blocos produzidos com substituição parcial de 50\% em volume do agregado miúdo mineral convencional pelo resíduo de Pinus spp. apresentaram características físicas e mecânicas que possibilitam sua utilização em alvenarias estruturais, para a construção de edifícios de até seis pavimentos;

A utilização do resíduo de Pinus spp. como substituto ao agregado miúdo convencional reduziu a condutividade térmica média do concreto produzido, o que proporciona reflexos importantes para o desempenho dos blocos quanto ao conforto térmico dos ambientes construídos.

\section{REFERÊNCIAS BIBLIOGRÁFICAS}

AMERICAN SOCIETY FOR TESTING AND MATERIALS - ASTM.ASTM C 1170 : Standard test method for determining consistency and density of roller-compacted concrete using a vibrating table, Pennsylvania, 2008.

AMERICAN SOCIETY FOR TESTING AND MATERIALS - ASTM.ASTM C 177: Standard test method for steady-state heat flux measurements and thermal transmission properties by means of the guarded-hot-plate apparatus, Pennsylvania, 2008.

ASSOCIAÇÃOBRASILEIRADAINDÚSTRIADE MADEIRA PROCESSADA MECANICAMENTE - ABIMCI. Estudo Setorial. Documento disponível em (http://www.abimci.com.br) >.Acesso em: 25 de setembro de 2009.

ASSOCIAÇÃO BRASILEIRA DE CIMENTO PORTLAND - ABCP. NBR 6467: Agregados Determinação do inchamento de agregado miúdo Método de ensaio. Rio de Janeiro, 2008.

ASSOCIAÇÃO BRASILEIRA DE CIMENTO PORTLAND - ABCP. NBR 7190: Projeto de estruturas de madeira. Rio de Janeiro, 1997.

ASSOCIAÇÃO BRASILEIRA DE CIMENTO PORTLAND - ABCP. NBR 7211: Agregados para concreto - Especificação. Rio de Janeiro, 2009.

ASSOCIAÇÃO BRASILEIRA DE CIMENTO PORTLAND - ABCP. NBR 7218: Agregados - Determinação do teor de argila em torrões e materiais friáveis. Rio de Janeiro, 1987.

ASSOCIAÇÃO BRASILEIRA DE CIMENTO PORTLAND - ABCP. NBR NM 248: Agregados Determinação da composição granulométrica. Rio de Janeiro, 2003.

ASSOCIAÇÃO BRASILEIRA DE CIMENTO PORTLAND - ABCP. NBR NM 30: Agregado miúdo - Determinação da absorção de água. Rio de Janeiro, 2001.

ASSOCIAÇÃO BRASILEIRA DE CIMENTO PORTLAND - ABCP. NBR NM 46: Agregados Determinação do material fino que passa através da peneira $75 \mu \mathrm{m}$, por lavagem. Rio de Janeiro, 2003.

ASSOCIAÇÃO BRASILEIRA DE CIMENTO PORTLAND - ABCP. NBR NM 49: Agregado miúdo - Determinação de impurezas orgânicas. Rio de Janeiro, 2001.

ASSOCIAÇÃO BRASILEIRA DE CIMENTO PORTLAND - ABCP. NBR NM 53: Agregado graúdo - Determinação da massa específica, massa específica aparente e absorção de água. Rio de Janeiro, 2003.

ASSOCIAÇÃO BRASILEIRA DE NORMAS TÉCNICAS - ABNT. NBR 12118: Blocos vazados de concreto simples para alvenaria - Métodos de ensaio. Rio de Janeiro, 2008.

ASSOCIAÇÃO BRASILEIRA DE NORMAS TÉCNICAS - ABNT. NBR 15220-4: Desempenho térmico de edificações. Rio de Janeiro, 2005.

ASSOCIAÇÃO BRASILEIRA DE NORMAS TÉCNICAS - ABNT. NBR 5733: Cimento Portland de alta resistência inicial. Rio de Janeiro, 1991.

ASSOCIAÇÃO BRASILEIRA DE NORMAS TÉCNICAS - ABNT. NBR 5738: Concreto Procedimento para moldagem e cura de corpos-deprova. Rio de Janeiro, 2003.

ASSOCIAÇÃO BRASILEIRA DE NORMAS TÉCNICAS - ABNT. NBR 6136: Blocos vazados de concreto simples para alvenaria - Requisitos. Rio de Janeiro, 2008.

ASSOCIAÇÃO BRASILEIRA DE NORMAS TÉCNICAS - ABNT. NBR 7215: Cimento Portland - Determinação da resistência à compressão. Rio de Janeiro, 1997.

ASSOCIAÇÃO BRASILEIRA DE NORMAS TÉCNICAS - ABNT. NBR 9479: Argamassa e concreto - Câmaras úmidas e tanques para cura de corpos-de-prova. Rio de Janeiro, 2006.

ASSOCIAÇÃO BRASILEIRA DE NORMAS TÉCNICAS - ABNT. NBR 9778: Argamassas e concretos endurecidos - Determinação da absorção 
de água, índice de vazios e massa específica. Rio de Janeiro, 2005.

ASSOCIAÇÃO BRASILEIRA DE NORMAS TÉCNICAS - ABNT. NBR NM 52: Agregado miúdo - Determinação da massa específica e massa específica aparente. Rio de Janeiro, 2003.

FAGUNDES. H. A. V. Produção de madeira serrada e geração de resíduos do processamento da madeira de florestas plantadas no Rio Grande do Sul. 2003. 173 f. Dissertação (Mestrado em Engenharia Civil) - Universidade Federal do Rio Grande do Sul, Porto Alegre, 2003.

FERREIRA JÚNIOR, S. Produção de Blocos de Concreto para Alvenaria; Prática Recomendada. Boletim Técnico ABCP, n. 107, 1985, 12 p.

FREITAS, L.C. A baixa produtividade e o desperdício no processo de beneficiamento da madeira: Um estudo de caso. 2000. $110 \mathrm{f}$. Dissertação (Mestrado em Construção Civil) -Universidade Federal de Santa Catarina, Florianópolis, 2000.

HACHMI, M.; CAMPBELL, A. G. Wood-cement chemical relationships. In: $1^{\text {st }}$ Inorganic Bonded Fiber Composites Symposium. Forest Products Research Socirty.Proceedings. P. 43-47. 1989.

HON, D.N.S; SHIRAISHI, N. Wood and Cellulosic Chemistry. NewYork: Marcel Dekker, Inc. 2001. $914 \mathrm{p}$.

ISAIA, G. C. Materiais de construção civil e princípios de ciência e engenharia de materiais. São Paulo: IBRACON. 2007. 1712 p.

IWAKIRI, S. Painéis de madeira reconstituída. Curitiba: FUPEF. 2005. 247 p.

JORGE, F. C.; PEREIRA, C.; FERREIRA, J. M. F. Wood-cementcomposites: a review. Holz als RohWerkstoff, n. 62, p. 370-377, 2004.

LIMA, A. F.; JARÁ, E. R. P.; ALFONSO, V. A. Madeira como matéria-prima para fabricação de pasta celulósica. In: Celulose e Papel: tecnologia de fabricação da pasta celulósica. São Paulo: IPT, 1988, p. 129-167.

MEDEIROS, J. S. Alvenaria estrutural não armada de blocos de concreto: Produção de componentes e parâmetros de projeto. 1993. 449 f. Dissertação (Mestrado em Engenharia Civil) Escola Politécnica da Universidade de São Paulo
São Paulo, São Paulo, 1993.

MORAIS, S. A. L.; NASCIMENTO, E. A.;MELO, D. C. Análise da madeira de Pinus oocarpa. Parte I - Estudo dos constituintes macromoleculares e extrativos voláteis. Revista Árvore, Viçosa, v. 29, n. 3, p. 461-470, 2005.

MOSLEMI. A. A. Wood-cement panel products: coming of age. In: INORGANIC BONDED FIBER COMPOSITES SYMPOSIUM.FOREST PRODUCTS RESEARCH SOCIETY. 1., 1988, Estados Unidos. Proceedings ... 1989, p. 12-18. RAMIREZ-CORETTI, A.; ECKELMAN, C. A.; WOLF, R. W. Inorganic-bonded composite wood panel systems for low-cost housing: a Central American perspective. Forest Products Journal, Madison, n. 48, p. 62-68, 1998.

SAVASTANO JÚNIOR, H., AGOPYAN, V. Microestrutura $\mathrm{x}$ desempenho dos compósitos reforçados com fibras vegetais. In: WORKSHOP SOBRE RECICLAGEM E REUTILIZAÇÃO DE RESÍDUOS COMO MATERIAL DE CONSTRUÇÃO CIVIL, SÃO PAULO, 1996, São Paulo. Anais. São Paulo, Epusp/Antac, 1997. p. 153-158.

SEMPLE, K. E.; EVANS, P. D. Wood-cement composites - Suitability of Western Australian mallee eucalypt, blue gum and melaleucas. Rural Industries Research and Development Corporation. Kingston: ACT, 2004. 64 p.

SIMATUPANG, M.H. et al. Influence of wood species on the setting of cement and gypsum. INTERNATIONAL CONGRESS ON FIBER AND PARTICLEBOARD WITH INORGANIC BINDER, 1988, Idaho. Proceedings... Idaho, 1988. p. 33-42.

SOUSA, J. G. G. Contribuição ao estudo da relação entre propriedades e proporcionamento de blocos de concreto - aplicação ao uso de entulho como agregado reciclado. 2001. 120 f. Dissertação (Mestrado em Engenharia Civil) - Universidade de Brasília, Brasília, 2001.

THE LEADING ASSOCIATION FOR THE WORLDWIDE PULP, PAPER, PACKAGING AND CONVERTING INDUSTRIES - TAPPI. TAPPI Cassical Method T 204 cm: Solvent extractives of wood and pulp, Georgia, 1997. 